Eur. Phys. J. AP 7, 277-289 (1999)

\title{
Experimental and numerical tools for miscible fluid displacements studies in porous media with large heterogeneities
}

\author{
P. Berest ${ }^{\mathrm{a}}$, N. Rakotomalala, J.P. Hulin, and D. Salin \\ Laboratoire Fluides, Automatique et Systèmes Thermiques ${ }^{\mathrm{b}}$, bâtiment 502, Campus Paris-Sud, 91405 Orsay Cedex, France
}

Eur. Phys. J. AP 6, 309-321 (1999)

Due to technical errors, the figures have been badly printed. We publish entirely the article herein, sincerely apologizing to the authors for the unpleasant inconvenience.

Received: 14 October 1998 / Revised: 17 February 1999 / Accepted: 24 March 1999

\begin{abstract}
We present a set of complementary experimental and numerical tools for studying miscible fluid displacements in porous media with large scale heterogeneities. Experiments are realized in transparent 2D Hele-Shaw cells allowing optical observations and in 3D packings of glass beads with an acoustical technique for imaging fluid displacements. Permeability heterogeneities are modeled by spatial variations of either the local aperture of the Hele-Shaw cell or the diameter of the grains used in the packing. The Hele-Shaw cell model provides high resolution maps of the invasion front location at regular time intervals and of the flow lines: the velocity field is determined by combining these informations. Acoustical images of relative concentration distributions in the 3D packing are in agreement with Hele-Shaw cell data and can be obtained in a broader range of experimental situations. Such experiments realized with a stabilizing density contrast between invading and displaced fluids demonstrate a strong reduction of the front width at low flow velocities, a similar reduction is obtained at high velocities with a stabilizing viscosity contrast. The technique is also applicable to study fluid displacements in natural opaque media. Numerical simulations by a Boltzmann lattice technique using a Stokes-like diffusive term to smooth out the effect of permeability discontinuities provide complementary informations. They are shown to give similar results as experiments for same flow parameter values and to allow for a fast exploration of a broad range of fluid properties and flow situations.
\end{abstract}

PACS. 47.55.Mh Flows through porous media $-43.58 .+\mathrm{z}$ Acoustical measurements and instrumentation - 47.55.-t Nonhomogeneous flows

\section{Introduction}

Heterogeneities of porous media are a key factor in many practical applications in such fields as petroleum, civil or chemical engineering or in the prediction of the dispersion of various types of wastes. However, such processes as oil recovery or waste dispersion depend little on small scale heterogeneities acting at the pore level: they are mostly influenced by large scale heterogeneities such as parallel strata [1-3] or channels [4-6] of varying permeabilities or large blocks of homogeneous internal structure but with

\footnotetext{
a e-mail: berest@ariane.fast.u-psud.fr

b UMR 7608 associated to CNRS and to the Pierre et Marie Curie and Paris-Sud Universities.
}

transport properties varying at random from one block to another $[7,8]$. These may for instance create preferential flow channels which greatly enhance the velocity of pollutant dispersion or, on the contrary, low permeability zones where large amounts of residual oil will remain unexploited. Determining the corresponding permeability fields is therefore a most relevant challenge for all such problems.

Since the permeability heterogeneity issue addresses flow in porous media, a natural approach to detect these heterogeneities is to monitor the motion of fluids inside them. Such a practical method for determining an heterogeneous porosity and permeability field is to follow the deformation of a "passive" displacement front of a fluid 
by another one of same density and viscosity. In a first approach, one can consider that the progression of the front follows the easiest flow paths in high permeability regions. Locally, the fluid superficial velocity $\mathbf{v}$ (defined from the flow per unit area) is related to the pressure gradient by Darcy's law:

$$
\mathbf{v}=-\frac{k}{\eta} \operatorname{grad} p
$$

where $\eta$ is the fluid viscosity and $k$ the space dependent permeability. If the fluid is incompressible, one has the additional relation:

$$
\operatorname{div} \mathbf{v}=0
$$

Let us now displace a transparent fluid by a dyed one of similar density and viscosity and determine at regular small time intervals $\Delta t$ the contour of the displacement front (given for instance by an implicit equation $\mathcal{F}(\mathbf{r}, t)=0)$; the velocity components normal to the front can be determined from the function $\mathcal{F}(\mathbf{r}, t)$ and its derivatives with respect to time and space coordinates. One can then show [9] that equations $(1,2)$ can be used to solve the inverse problem of the computation of the permeability distribution $k(\mathbf{r})$ from time variations of the displacement front. For that purpose, it is first necessary to devise methods allowing to determine the front profiles at regular time intervals, even in opaque and $3 \mathrm{D}$ systems. Then, one needs to analyze quantitatively the relation between the displacements of these profiles and the local permeability distribution.

As a first step towards this goal, we report in the present paper a study by complementary methods (one numerical and two experimental) of concentration distribution variations in model heterogeneous media constructed from a few blocks of different permeabilities. Both types of experiments are performed by displacing a fluid by another miscible one of same or different viscosity. The experiments and numerical simulations are realized with the same geometry of permeability heterogeneities.

The first model experimental system uses a transparent Hele-Shaw cell with a variable local thickness reproducing permeability changes. This setup allows to perform direct visual and video observations, to determine through an image analysis procedure time variations of the displacement front and finally to obtain the velocity field.

A second experimental system uses a 3D packing of grains: permeability variations are obtained by varying locally the diameter of the packed grains. In this opaque material, measurements are performed by an ultrasonic scanning technique providing an acoustic image of the fluid distribution in the medium. These 3D measurements have a lower resolution than $2 \mathrm{D}$ visualizations but allow more easily to study the influence of density and viscosity contrasts on fluid displacement by using different fluids. This technique has been applied in the present work to permeability distributions invariant along one of the directions perpendicular to the flow: in this case, results are directly comparable to Hele-Shaw cell experiments. On the other hand, such measurements can also be realized on fully side cut view

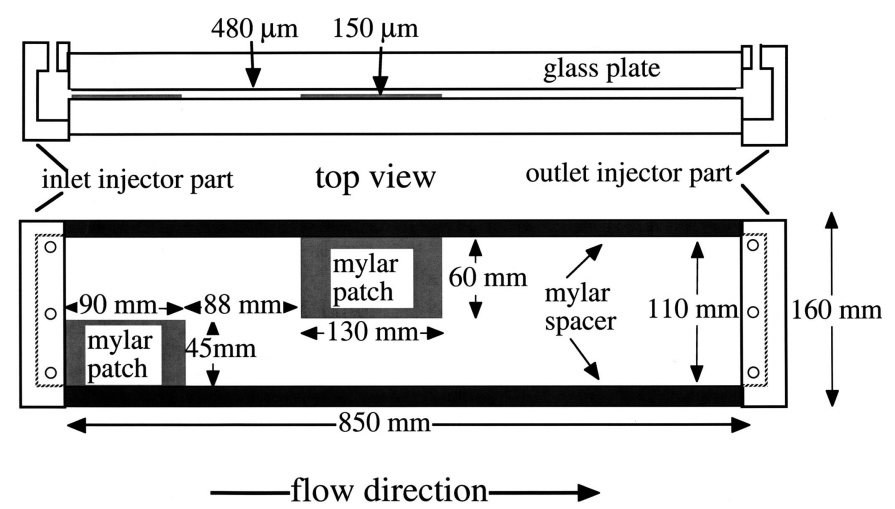

Fig. 1. Schematic view of the Hele-Shaw cell experiment.

3D systems and natural materials in which permeability varies in all directions.

The experimental results are finally compared to numerical simulations of fluid displacements realized with a Boltzmann lattice algorithm modified to take into account discontinuities of the spatial permeability distribution. Once their validity has been verified, these simulations provide a flexible and fast approach to investigate the influence of the fluid characteristics and of the distribution and spatial correlation of permeability variations.

\section{Hele-Shaw cell experiments}

\subsection{Experimental setup and procedure}

The Hele-Shaw cell device is realized with two parallel $1 \mathrm{~cm}$ thick glass plates with a length of $850 \mathrm{~mm}$ and a width of $160 \mathrm{~mm}$. The two plates are horizontal and separated by $480 \mu \mathrm{m}$ thick mylar spacers parallel to their long sides and leaving a $110 \mathrm{~mm}$ wide channel in the center (Fig. 1). In the Hele-Shaw cell approximation, pressure is uniform in the direction perpendicular to the plates and the mean local velocity $\mathbf{V}_{\mathrm{m}}$ averaged over the aperture verifies:

$$
\mathbf{V}_{\mathrm{m}}=-\frac{h^{2}}{12 \eta} \operatorname{grad} p
$$

This equation represents the 2D equivalent of Darcy's law (1) in which the equivalent local permeability $k$ is equal to $h^{2} / 12$. Permeability variations can therefore be mimicked by varying locally the distance between plates: this is achieved practically by gluing patches of mylar sheets onto the glass surface. Their geometry is described in more detail below.

An injector part is pressed against the inlet end (parallel to the width of the plates): it includes a parallelepipedic slot with a $6 \times 10 \mathrm{~mm}$ cross-section and a length of $120 \mathrm{~mm}$ secured across the full width of the flow channel. Fluid is injected through 3 tubes located at both ends and at the middle of the slot. In the initial phase, the setup 
is fully saturated with transparent fluid. The injector part is then saturated with dyed fluid by circulating it between the three injection tubes (the outlet valve located at the other end of the flow channel is closed). Finally all inlet tubes are connected in parallel to an injection syringe pump and the outlet valve is opened. This procedure allows to get a straight uniform dye concentration variation front penetrating at the origin time into the model. The fluid flow rate is measured by computer controlled scales and ranges between 5 and $200 \mathrm{ml} / \mathrm{h}$ which corresponds to typical mean flow velocities between $2.5 \times 10^{-2}$ and $1 \mathrm{~mm} / \mathrm{s}$ (or Peclet numbers $P e=V_{\mathrm{m}} h / D_{\mathrm{m}}$ ranging between 35 and 1500 in the uniform thickness parts of the model, $D_{\mathrm{m}}$ is the molecular diffusion coefficient of the dye).

The fluids used are water-glycerol solutions of viscosity $5.4 \times 10^{-3} \mathrm{Pas}$. One of the solutions is dyed by dissolving $1 \%$ of eosine in weight. $1.44 \mathrm{~g} / \mathrm{l}$ of cadmium chloride $\left(\mathrm{CdCl}_{2}\right)$ are dissolved into the other - transparent - solution so that that the two fluids have matched densities. The typical value of $D_{\mathrm{m}}$ for the dye is $3 \times 10^{-10} \mathrm{~m}^{2} / \mathrm{s}$. Some local density contrasts between the dyed and transparent fluids may however appear, for instance due to unequal mutual diffusion of eosine and $\mathrm{CdCl}_{2}$ between the two solutions. Using fluids of higher viscosity than plain water allows to minimize their influence that might be particularly significant, - even for an horizontal flow - in the Hele-Shaw device. The local aperture of the flow channels (the local spacing between plates) is indeed higher than in usual porous media: this enhances gravity driven convection flows that increase very fast with the channel size.

In the Hele-Shaw cell experiment, the broadening of the concentration variation front results from the combination of two factors. One is spreading by velocity gradients parallel to the glass plates and due to spatial variations of the local thickness: this represents the permeability heterogeneity effect we wish to study. A second - more local - mechanism is spreading due the Poiseuillelike parabolic velocity profile between the plates. This dispersion effect is moderated by molecular diffusion perpendicular to the plates resulting in the well-known Taylor dispersion mechanism [10].

For a cell of uniform thickness and particle transit times long enough compared to the diffusion time across the aperture, one reaches a Gaussian dispersion regime; then, the average concentration across the cross-section verifies the classical one dimensional convection-diffusion equation $[1,10]$ :

$$
\frac{\partial C}{\partial t}+\mathbf{V}_{\mathrm{m}} \cdot \operatorname{grad} C=D_{\|} \frac{\partial^{2} C}{\partial x_{\|}^{2}}
$$

in which the index $\|$ corresponds to the direction of the mean flow velocity $\mathbf{V}_{\mathrm{m}}$. The mean concentration is advected at the velocity $\mathbf{V}_{\mathrm{m}}$ while the front spreads out with the longitudinal dispersion coefficient $D_{\|}$verifying the Taylor-Aris equation $[10,11]$ :

$$
D_{\|}=D_{\mathrm{m}}+\frac{h^{2} V_{\mathrm{m}}^{2}}{210 D_{\mathrm{m}}} .
$$

The typical concentration variation front width after a path length $L=V_{\mathrm{m}} t$ is $\Delta x=\sqrt{2 D_{\|} t}$. In our experiments, $\Delta x$ must be minimized since no front spreading mechanism of the same kind is encountered in $3 \mathrm{D}$ porous media. $\Delta x$ is found easily to be minimal and of order $\Delta x_{\min } \approx \sqrt{L h / 3.5}$ for a Péclet number value $P e_{\min }=$ $h V_{\mathrm{m}} / D_{\mathrm{m}} \cong 15$. For $L=900 \mathrm{~mm}$ and $h=0.48 \mathrm{~mm}$, this gives $\Delta x_{\min } \cong 11 \mathrm{~mm}$ which, in the present experiments, is markedly smaller than the contribution of permeability heterogeneities of interest to us. $P e_{\min }$ corresponds to a velocity of about $10^{-3} \mathrm{~cm} / \mathrm{s}-$ a factor of 2.5 lower than the smallest velocity we used. At the highest flow-rates used in our work, $\Delta x$ would be of the order of $7 \mathrm{~cm}$.

\subsection{Experimental measurements and analysis of Hele-Shaw cell experiments}

The experimental cell is horizontal and uniformly illuminated from below. A video camera is placed at a height of $1670 \mathrm{~mm}$ above the plates and a $28 \mathrm{~mm}$ focal length lens provides a field of view of $380 \times 126 \mathrm{~mm}$. The output of the video camera is directly digitized and stored on a computer and 120 images are recorded for each displacement sequence. All experiments described in this paper have been realized with the following permeability distribution: the local thickness of the Hele-Shaw cell is everywhere uniform and equal to $e_{+}=480 \mu \mathrm{m}$ but for two rectangular zones of respective dimensions $44 \times 90 \mathrm{~mm}$ and $66 \times 130 \mathrm{~mm}$ (Fig. 1) where it is reduced to $e_{-}=305 \mu \mathrm{m}$. This correspond to a permeability ratio $k_{+} / k_{-}=\left(e_{+} / e_{-}\right)^{2} \cong 2.48$ between high and low permeability regions. Figures $2 \mathrm{a}$ and $2 \mathrm{~b}$ display the distribution of dye in the experimental cell at two times $t=\Delta t$ and $t=3 \Delta t$ after the beginning of the injection $(\Delta t=900 \mathrm{~s})$. At short times (Fig. 2a), the dye front moves - as expected - slower in the low permeability region (bottom left) than in the rest of the cell. At longer times (Fig. 2b), a preferential path inside which the tracer concentration front is markedly ahead of the other parts of the flow is clearly visible.

Once images of the invasion of the Hele-Shaw cell by coloured fluid have been obtained, they are first analyzed by determining the line contour of the dyed zone. The first image of the series corresponds to the initial situation in which no dye has penetrated yet into the cell; a second reference image corresponds to the final situation of a cell fully invaded with dyed fluid at the concentration of the injected solution. All images (including the final state) are then subtracted from the first one and normalized thereafter by the corrected final one. This allows, in particular, to compensate - at least approximately - image density changes due to thickness variations. The result is a set of numbers between 0 and 1 which is multiplied by 255 to allow visualization by image processing programs; the image is finally binarized by thresholding at the median value 127 and the front is determined after applying a noise reduction algorithm to eliminate stray transitions.

Figure 3a displays the contour of the fronts at 9 different acquisition times separated by regular intervals $\Delta t=300 \mathrm{~s}$ : the development of a finger-like structure 

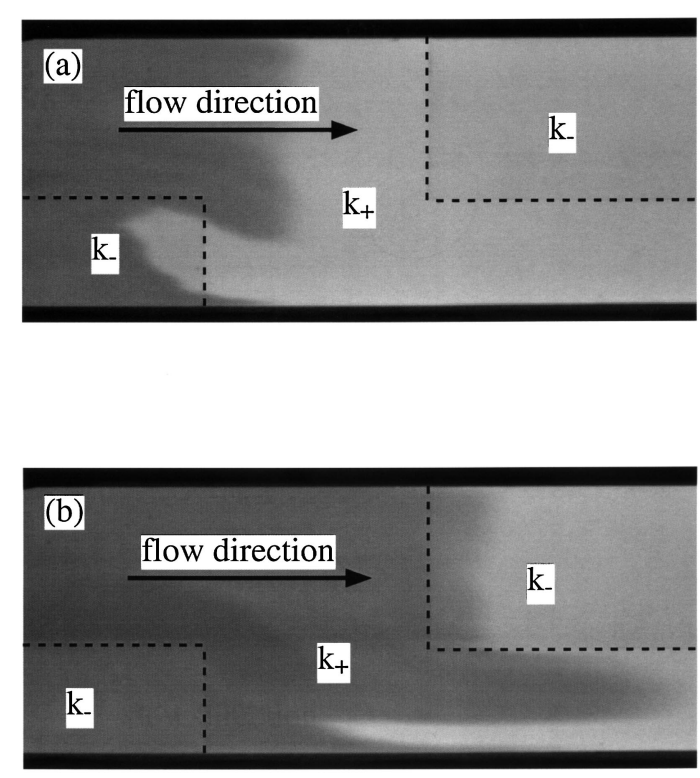

Fig. 2. View from above of the experimental Hele-Shaw cell during the displacement of a transparent fluid (light colour) by a dyed one (dark colour). Mean fluid velocity in constant thickness sections $=2.5 \times 10^{-2} \mathrm{~mm} / \mathrm{s}(P e=35)$. (a) Time lapse $t=1500 \mathrm{~s}$ after the beginning of the injection. Dotted lines correspond to the boundaries of the two rectangular low permeability zones in the sample; (b) $t=4500 \mathrm{~s}$. Flow is from left to right.

is fast flow paths is clearly observed as well as strong distortions at the boundaries of low permeability zones. These reflect the fact that velocity is discontinuous at the boundaries, particularly far enough from their upstream edge when flow lines are again parallel to the length of the model: the longitudinal pression gradient is continuous across the boundary so that the ratio of the velocities in both zones reflects the ratio of the permeabilities.

In order to obtain more quantitative informations, it is now necessary to determine the velocity field from these experiments. It would be possible, in principle, to determine directly the flow lines and the fluid velocity field from front contours by simple mass conservation considerations (taking into account the fact that the side boundaries of the model must be flow lines). The fluid volume delimited by any two given flow lines and two concentration fronts determined at times $t$ and $t+\Delta t$ must indeed be constant with $t$ for a stationary flow (if $\Delta t$ is kept the same). However, the computation is not straightforward since this volume is also proportional to the local thickness for given front and flow lines: the thickness value should be input a priori in the computation and adjusted to match the measurements and verify the flow equations. Such a complex procedure would introduce large additional uncertainties.

We have therefore determined the streamlines by independent measurements displayed in Figure 3b. Dye is injected in this case at a selected point: since flow is stationary, the emission lines observed are identical to the corresponding streamlines. The experiment is repeated at
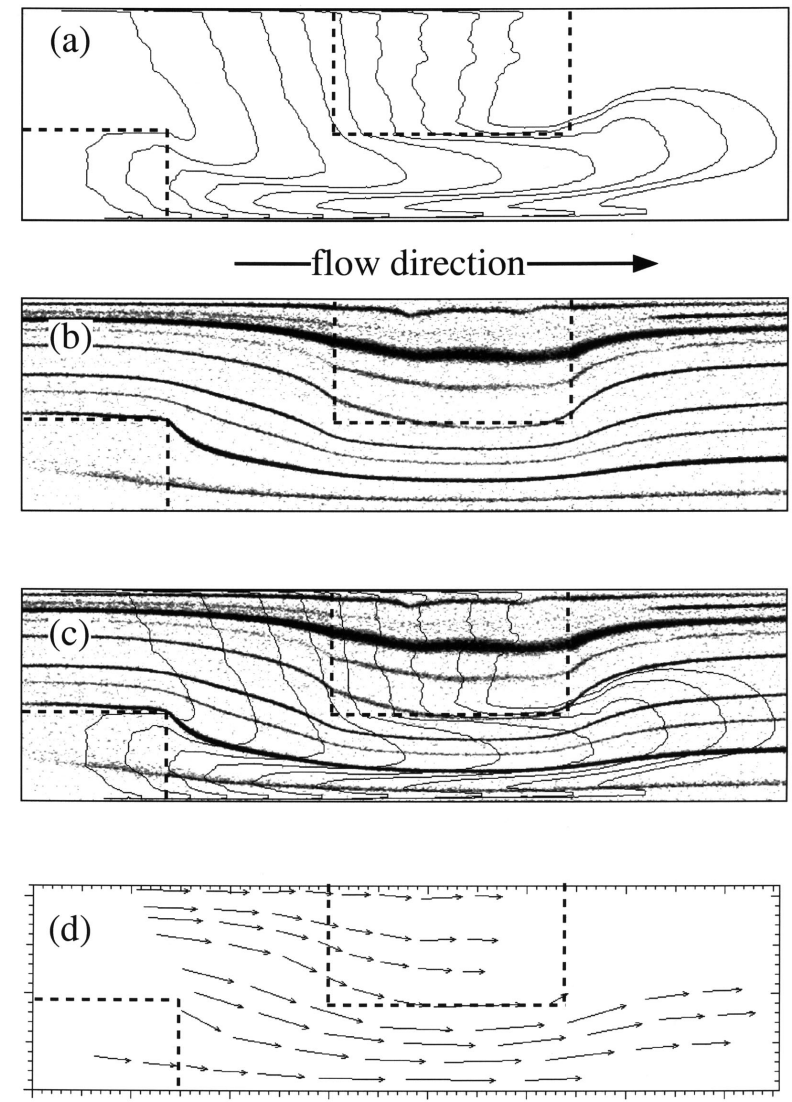

Fig. 3. Interpretation of concentration distribution and emission lines measurements in invasion experiments performed at the same velocity and with the same fluids as in Figure 2. (a) Sequence of tracer invasion fronts obtained at regular time intervals $\Delta t=450 \mathrm{~s}$ during the displacement of a transparent fluid by the same dyed one. (b) Flow lines visualized by localized injection of tracer at selected points. (c) Superposition of invasion fronts and flow lines. (d) Estimation of velocity vector field from (c).

several injection points (one at a time to minimize perturbations of the flow field). Since flow is laminar in the experiment, this measurement does not need to be repeated for all flow rate values: the streamlines remain the same although the modulus of the velocity vector is proportional to the flow rate. It can be seen that dyed streaks become thicker inside the low permeability zone at the center of the image: this is due to the fact that they are bounded by flow lines which move apart to insure flow-rate conservation between them when velocity decreases.

The streamlines and invasion front maps can then be superimposed (Fig. 3c). This allows to determine directly the velocity field if the various lines are spaced sufficiently closely: the local velocity vector is indeed nearly parallel to the segment joining the intersections of the streamlines with two subsequent concentration fronts. Its modulus can be computed from the distance between intersections. Figure $3 \mathrm{~d}$ displays the velocity vector field obtained in this way from Figure 3c. 
It is interesting, in particular, to compare flow velocities measured in this way in high and low permeability zones: we looked particularly at the center part of the field of view in Figures $3 \mathrm{~b}$ and $3 \mathrm{c}$ where streamlines are sufficiently closely parallel so that one can assume that pressure gradient parallel to the mean flow is the same in both regions. The measured velocity ratio between both zones is of the order of 2.5-3 which is in reasonable agreement with the permeability ratio of 2.48 estimated from aperture thickness values in both regions.

The velocity field determined experimentally in this way has been compared to computation results obtained using a lattice gas dynamics algorithm to be described in detail in Section 4. Figure 10 displays concentration fronts, flow lines and velocity fields computed numerically for a set of flow parameters and for a cell-geometry similar to that used experimentally. One observes that the sets of curves in Figures 3 and 10 are qualitatively extremely similar.

\section{Experiments on 3D packings}

Hele-Shaw cell experiments described above provide a detailed determination of the distortion of miscible displacement front in porous media containing a $2 \mathrm{D}$ array of blocks of different permeabilities. Experiments performed on glass bead packings with a similar spatial permeability distribution will now allow us to check the validity of this experimental model to the description of 3D systems. This set-up allows - in addition - to analyze the part played by density and viscosity contrasts between the displacing and displaced fluids. Finally, it is also possible to analyze fully 3D systems in which permeability varies in all directions.

We realized these measurements with an acoustical scanner device providing a $10 \times 17$ resolution image of the fluid distribution from sound velocity measurements. This technique is applicable to non transparent materials such as rocks and, for transparent materials, it does not require index matching fluids like optical techniques. The technical complexity and cost is also lower by several orders of magnitude than that of more sophisticated devices like X-ray CAT scanners or NMR images. While the spatial resolution is not as good as that of such techniques, the precision on the relative concentration values is comparable and, in some cases, better. Using this technique requires however that the porous medium be fully saturated with liquids to avoid attenuation due to gas. Also, the sound propagation path length must be shorter than about $10 \mathrm{~cm}$ in order to allow using frequencies high enough (typically $350 \mathrm{kHz}$ ) to give a good precision on concentration values.

\subsection{Experimental procedure and acoustical measurements}

Miscible displacement experiments are performed at different values of the flow rate $Q$, in a patchwork packing of glass beads of length, $L=300 \mathrm{~mm}$ in the vertical direc-

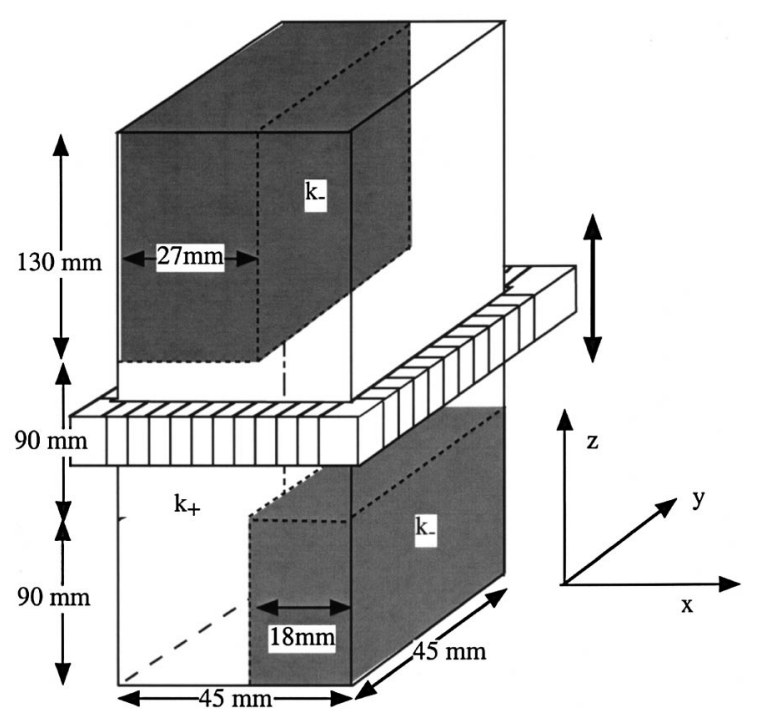

Fig. 4. Schematic view of experimental set-up for acoustic scanner measurements of invasion processes in heterogeneous $3 \mathrm{D}$ bead packings. The dark part corresponds to the zone where the permeability of the packing has been reduced by using smaller diameter beads.

tion, with an horizontal square cross-section $(45 \times 45 \mathrm{~mm})$, corresponding to an aspect ratio of the order of 7 (Fig. 4).

The 3D patchwork-like porous medium, of geometry identical to the Hele Shaw cell, is prepared by first partitioning the system into 2 vertical parallel compartments of respective widths 18 and $27 \mathrm{~mm}$, using a very thin vertical spacer plate. The two compartments are filled with successive layers of glass beads of diameters varying from layer to layer in order to create permeability heterogeneities. The horizontal interfaces between layers vary from one compartment to the next which allows to create sets of parallelepipedic heterogeneities with arbitrary locations and lengths parallel to the flow. The spacers are gently removed thereafter. In the samples used at present, the local permeability is constant along the horizontal direction $y$ parallel to the spacer plates: the permeability distribution is then bidimensional and the flow velocity field should be comparable to that observed in Hele-Shaw cell models. Flat sintered metal beads plates placed at the bottom and the top of the sample distribute evenly the flow rate across the flow section.

We have used a sample with a permeability distribution geometry nearly equivalent to that of the experiments of part 2 (Fig. 4). The sample includes two parallelepipedic regions prepared from glass beads of diameter $d_{1}=145 \pm 20 \mu \mathrm{m}$ while the remnant of the packing is filled with beads of diameter $d_{2}=285 \pm 35 \mu \mathrm{m}$. The permeability $k_{-}$in the two heterogeneities is then about 4 times lower than the value $k_{+}$in the rest of the sample (the local permeability varies roughly as the square of the bead diameters). One of the low permeability regions of dimensions $18 \times 45 \times 90 \mathrm{~mm}$ is located right at the inlet of the sample; it is separated by $90 \mathrm{~mm}$ (parallel to the flow) from the second one which is of dimensions $27 \times 45 \times 130 \mathrm{~mm}$ and extends up to the outlet section. 
Table 1. Characteristic of fluids used in displacements experiments in the heterogeneous porous packing.

\begin{tabular}{ccccccc}
\hline displaced fluid & injected fluid & $\mu_{-}(\mathrm{Pa} \mathrm{s})$ & $\mu_{+}(\mathrm{Pa} \mathrm{s})$ & $M=\mu_{+} / \mu_{-}$ & $\rho_{-}(\mathrm{g} / \mathrm{ml})$ & $\rho_{+}(\mathrm{g} / \mathrm{ml})$ \\
\hline $48 \%$ glycerol-water & $38 \%$ sucrose-water & $5.4 \times 10^{-3}$ & $5.3 \times 10^{-3}$ & 0.98 & 1.12 & 1.1663 \\
$32 \%$ sucrose-water & $54 \%$ glycerol-water & $3.75 \times 10^{-3}$ & $7.5 \times 10^{-3}$ & 2 & 1.1366 & 1.1364 \\
$54 \%$ glycerol-water & $32 \%$ sucrose-water & $7.5 \times 10^{-3}$ & $3.75 \times 10^{-3}$ & 0.5 & 1.1364 & 1.1366 \\
\hline
\end{tabular}

The mean flow is vertical and parallel to the length of the sample in order to avoid possible problems with gravity override $[12,13]$. Such problems are known to occur when gravity is perpendicular to the main flow direction: then, the shape of the displacement front often gets strongly distorted since the heavier fluid moves towards the lower part of the sample. The flowing fluids are mixtures of water and glycerol and/or of water and sucrose. It is possible to obtain various combinations of viscosity and density contrasts by varying the concentration of each fluid: in particular equal density-different viscosity, and equal viscosity-different density mixtures can be obtained (Tab. 1).

The concentration profile $C(x, y, z, t)$ is determined from measurements of the sound velocity which depends on the relative volumetric concentrations of the two fluids saturating the medium $[14,15]$. The acoustic scanner we are using measures, at a given vertical location $z$, the average transverse concentration along 10 horizontal propagation paths parallel to the $x$ direction and 10 paths parallel to the $y$ direction. 20 pairs of parallel transducers with a $4 \mathrm{~mm}$ spacing between successive propagation paths are needed for that purpose. From the calibration curve and the accuracy of the relative velocity measurements $\left(10^{-4}\right)$ we estimate that the precision on the value of the average concentration along a given path is generally of order $1 \%$. This precision may be degraded in some cases by acoustic artefacts such as interferences between the signal beam and refracted waves at a distorted interface ("mirage" like effect) or reflections on the side walls. The spatial resolution is close to the typical size of the transducers, namely $2 \mathrm{~mm}$ in the $x$ or $y$ horizontal directions and $1 \mathrm{~cm}$ along the vertical. The whole set of 20 transducer pairs can be displaced vertically along the column: in a first series of experiments we performed measurements in 13 cross-sections spaced by $15 \mathrm{~mm}$ and located at distances $z$ ranging from 22 to $202 \mathrm{~mm}$ from the inlet plate. At a given $z$ value, we measure 10 concentrations averaged along the $x$ direction and 10 along $y$ at regular intervals respectively along $y$ and $x$. The full cycle of measurements is then repeated periodically: the typical duration of each cycle is of the order of $120 \mathrm{~s}$. In a second series of experiments, measurements were performed in 17 cross-sections also spaced by $15 \mathrm{~mm}$ and located at distances $z$ between 22 and $262 \mathrm{~mm}$ from the inlet plate.

In the present case of a $2 \mathrm{D}$ permeability distribution invariant along the $y$ direction, sound velocity measurements with a propagation along $y$ allow to analyze variations of the concentration distribution across the heterogeneities. Measurements in the perpendicular direction average out the influence of permeability variations along $x$; they allow to check that the concentration front is actually bidimensional by verifying that $C(t)$ curves obtained at different distances $y$ for the same $x, z$ coordinates are identical.

Each experiment provides a set of 260 (or 340 for 17 cross-sections) time variations curves: these curves are analyzed directly or transformed into instantaneous images of the spatial concentration distribution. The latter are obtained by first extracting from the result files concentrations values $\overline{C(x, z, t)}$ corresponding to a chosen time $t$. Then these experimental curves are normalized between two limiting values obtained respectively before the displacement and at very long times at the same measurement point. Instantaneous concentration images with 256 grey levels and a resolution of $17 \times 10$ are finally reconstructed from these set of data: white and black colours correspond respectively to a complete saturation of the medium by the displaced (initial situation) and the displacing (final state) fluids. Although the spatial resolution is lower, the results of these acoustical scanner measurements can be easily compared to those of Hele-Shaw cell experiments.

\subsection{Experimental concentration variation measurements in bead packings}

Figures 5a and 5b display time concentration variation curves obtained in a same section of the medium of Figure 4 . The ratio $\rho_{+} / \rho_{-}$between the densities of the displacing and displaced fluid is 1.042: the configuration is stable so that one expects gravitational buoyancy effects to reduce the front width, particularly at low flow velocities. On the other hand, the viscosities $\mu_{+}$and $\mu_{-}$ of the injected and displaced fluid are nearly identical ( $M=\mu_{+} / \mu_{-}=0.98$ ) so that no influence of viscous effects on the displacement is expected. These experiments have been performed with flow velocities of order $7.5 \times 10^{-2} \mathrm{~mm} / \mathrm{s}$ in the constant permeability region: this corresponds to the upper end of our experimental range so that the influence of buoyancy effects is small and the concentration variation distribution is mostly determined by the structure of the flow field.

The curves of Figure 5a have been obtained for sound propagation parallel to axis $x$ at different transverse locations along $y$ : as expected, the curves overlay each other quite precisely which confirms the $2 \mathrm{D}$ structure of both the medium and the displacement flow (the measurement section is located between the two permeability heterogeneities). The contributions of zones of different 

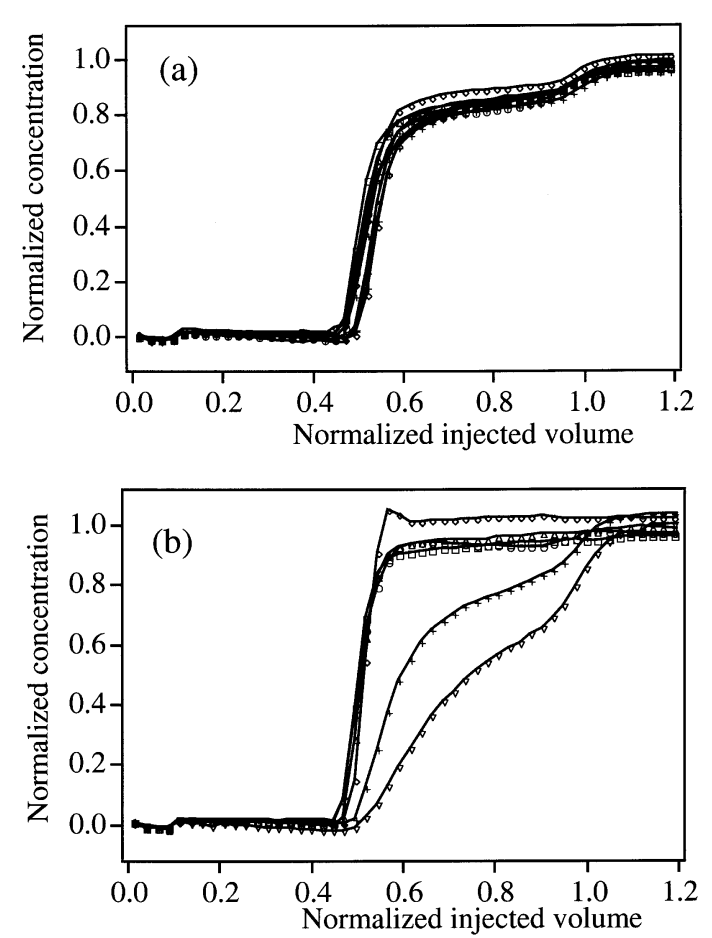

Fig. 5. Concentration variation measurements at three sections of the heterogeneous packing at various distances from the inlet (fluids of matched viscosity). (a) Time variation curves obtained for a propagation along $\bar{x}$ in a same section but at different transverse locations along $y$ (transducers are evenly spaced over a $36 \mathrm{~mm}$ interval inside the $45 \mathrm{~mm}$ width of the channels. (b) Time variation curves obtained in the same sample section for sound propagation along $y$ at different transverse distances along $x$ (using the same numbering as in Fig. 6, $(\diamond)$ corresponds to transducer \#2, $(\triangle) \# 5,(\square) \# 6,(\circ) \# 7,(+)$ $\# 9,(\nabla) \# 10)$. Flow rate $Q=200 \mathrm{ml} / \mathrm{h}\left(V=7.6 \times 10^{-2} \mathrm{~mm} / \mathrm{s}\right)$, $z=127 \mathrm{~mm}$ (counted from sample inlet and corresponding to plane 8 in Fig. 6), $38 \%$ sucrose solution displacing $48 \%$ glycerol solution $\left(M=\mu_{+} / \mu_{-}=0.98, \rho_{+} / \rho_{-}=1.04\right)$.

permeabilities are identified in these experimental curves from the observation of several fronts moving at different speeds.

On the other hand, the curves of Figure $5 b$ - still obtained in the same section - correspond to a propagation along $y$ at different distances along $x$ : significant differences are observed, resulting from distortions of the flow field due to the heterogeneities. Concentration variation fronts move at different speeds in various parts of the medium: these velocity contrasts are induced by permeability heterogeneities. One observes that the concentration variation is sharp for measurements points located on streamlines which have remained nearly parallel all along their path; it is broader for streamlines which have experienced lateral deviations and significant velocity gradients. This broadening of concentration variation curves can be observed experimentally because the transducers have a finite non-zero size; also, local dispersion effects allow fluid particles of the two species to explore velocity gradients which further broadens the tracer concentration variation
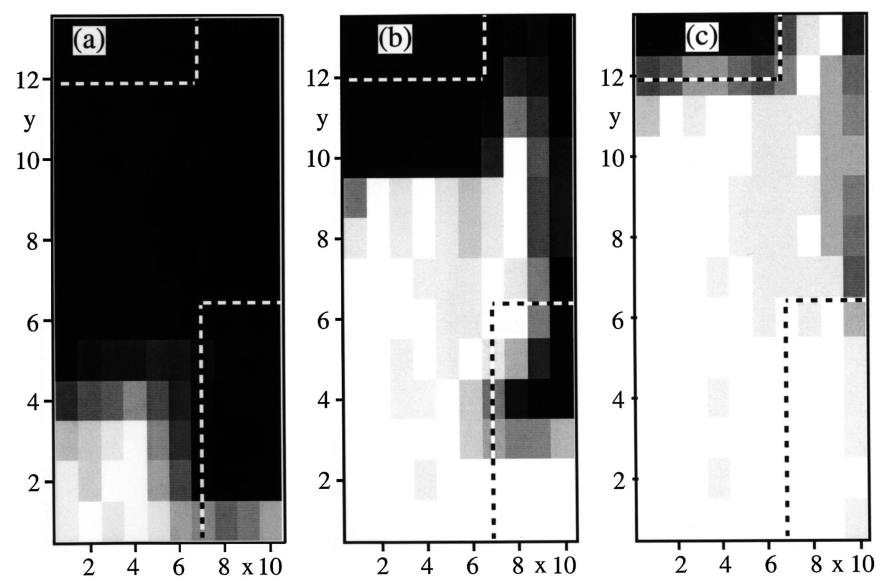

Fig. 6. Grey scale concentration variation images computed for the same experiment as Figures $5 \mathrm{a}$ and $5 \mathrm{~b}$ using measurements performed at 13 equally spaced distances $z$ from the inlet section. Flow rate $Q=200 \mathrm{ml} / \mathrm{h}\left(V=7.6 \times 10^{-2} \mathrm{~mm} / \mathrm{s}\right)$, $38 \%$ sucrose solution displacing $48 \%$ glycerol solution $(M=$ $\mu_{+} / \mu_{-}=0.98, \rho_{+} / \rho_{-}=1.04$ ); (a) normalized injected time $t_{\mathrm{r}}=$ injected volume $/$ pore volume $=0.35$; (b) $t_{\mathrm{r}}=0.65 ;$ (c) $t_{\mathrm{r}}=0.95$. The direction of the fluid displacement is upwards.

front. A few data points in Figures $5 \mathrm{a}$ and $5 \mathrm{~b}$ indicate erroneous relative concentration values larger than 1: this is due to acoustic artefacts of the type discussed in Section 3.1 .

Figures $6 \mathrm{a}-6 \mathrm{c}$ display a typical sequence of $10 \times 13$ pixel concentration images obtained for the same experiment as the curves of Figures $5 \mathrm{a}$ and $5 \mathrm{~b}$ and for several normalized injected volumes of displacing fluid. One observes clearly that the invasion of the low permeability regions is delayed with respect to that of high permeability channels (dotted lines mark the boundaries between the various zones). The low permeability region near the inlet of the sample gets saturated by the injected fluid only towards the end of the displacement process. Another observation is the fact that, during the displacement, heavier injected fluid particles moving through fast flow paths may reach a locally unstable configuration overhanging lighter fluid saturating initially the sample. This may result in a downward motion of these heavier fluid particles, particularly at low flow velocities.

Concentration distributions displayed in Figures 6a6c can be compared usefully to those reported above in the equivalent Hele-Shaw cell model (the fluid properties and flow velocity have been chosen so that both the influence of the density and of the viscosity contrasts are minimized). We observe first qualitatively that concentration distribution variations observed in Figures 2 and 6 are very similar: the essential feature of a fast penetration finger leaving on both sides residual displaced fluid is particularly well-reproduced. Thus, the main features of the invasion process are similar for the Hele-Shaw model and the heterogeneous bead packing.

Let us however analyze a few differences between these two experiments that may influence details of the concentration distribution. 
A first one is the fact that thickness variations allowing to adjust the local permeability of the Hele-Shaw cell also modify to a minor degree the fluid volume (the local "porosity"). On the contrary, in the 3D packing varying the grain size to change the permeability leaves the porosity almost unchanged. Thus, velocity fields in the two cases may be differ slightly even for same permeability distributions.

A second issue is the difference between the local mechanisms broadening the concentration variation fronts at small scales. In the Hele-Shaw cell, the mechanism is Taylor dispersion already discussed in Section 2.1; in the second case, one deals with geometrical dispersion due to the disordered structure of the packings at the grain scale. The corresponding local geometric dispersion coefficient is of the order of the product of the flow velocity and the typical grain size $d_{\mathrm{g}}$ [16-18]. The corresponding thickness of the local mixing region between the invaded and displaced fluid after a path length $L$ is of order $\sqrt{L d_{\mathrm{g}}}$ which is less than a cm. The influence of either local mechanism on front spreading is then almost always much smaller than that of the velocity contrasts between blocks of different permeabilities $[7,8]$. This is particularly the case in the Hele-Shaw cells or bead packings we are using where large heterogeneities induce strong velocity variations.

The mechanism of transverse exchange between flow lines is also different in the two systems. It is purely due to molecular diffusion in the Hele-Shaw cell and markedly enhanced by the disorder of the flow field in the bead packing. Finally, the influence of gravity differs in the horizontal Hele-Shaw cell and in the vertical bead packing where it may be felt even for very low density contrasts. Let us now analyze this effect in the bead packing as well as that of the viscosity contrasts.

\subsection{Influence of density and viscosity contrasts between the two fluids}

We have studied the influence of the contrast between the properties of the displacing and displaced fluids by performing displacement experiments with different fluid pairs. The characteristics of the displacement process are determined by three key control parameters: the mobility ratio, $M=\mu_{+} / \mu_{-}$denotes the ratio of the viscosity of the displaced fluid (subscript + ) divided by that of the displacing fluid (subscript -); the gravity number, $N_{\mathrm{g}}=\left(\rho_{+}-\rho_{-}\right) K g / \mu_{+} V$, is a dimensionless measurement of the relative influence of the buoyancy difference compared to the viscous effects and the Peclet number $P e=V d_{\mathrm{g}} / D_{\mathrm{m}}$ characterizes the relative magnitude of convective and diffusive mass transport. Here, $g$ denotes the component of gravity in the flow direction $z, K$ is the arithmetic average of the local permeabilities, $V$ is the average flow velocity across the sample section (always assumed to be positive), $D_{\mathrm{m}}$ is the molecular diffusion coefficient (typ. $1.5 \times 10^{-10} \mathrm{~m}^{2} / \mathrm{s}$ and $d_{\mathrm{g}}$ is the average grain size. In the experiments, $V$ varies over one decade, from $1.8 \times 10^{-3}$ to $7.2 \times 10^{-2} \mathrm{~mm} / \mathrm{s}$ (the corresponding Péclet numbers $P e$ range between 1.5 and 60 ).
Viscosity and density contrasts between the displacing and displaced fluids are a particularly important factor since they may result in instabilities of the displacement front at some values of the flow velocity. The onset of instabilities in homogeneous (one-layer) systems due to density and/or viscosity contrasts between the two fluids has been discussed in a previous work [19-21]: it has been shown in particular that viscous-unstable displacements $(M>1)$ can be stabilized by gravity $\left(N_{\mathrm{g}}<0\right)$, and vice versa. Specifically, the front is stable with respect to long wave perturbations as long as the Saffman-Taylor-Hill condition [22], $M-1+N_{\mathrm{g}} M<0$ holds.

In order to increase and analyze the influence of gravity, we have realized a series of experiments with the same couple of fluids as for Figure 5 (viscosity ratio $M$ equal to 1) but at lower fluid velocities. The corresponding variations of the average concentration at three different distances from the inlet are displayed in Figure 7 as a function of the injected volume (normalized by the total pore volume of the sample). The gravity number $N_{\mathrm{g}}$ as defined as above varies between $2 \times 10^{-2}$ in Figure $7 \mathrm{a}$ and 0.4 in Figure $7 \mathrm{c}$ (in the experiments of Fig. $5, N_{\mathrm{g}}$ is of the order of $10^{-2}$ ); the effect of gravity is always stabilizing since the heaviest fluid (the water-sucrose solution) is below the lighter water-glycerol solution. The influence of gravity is very clear and concentration variation fronts are much narrower and sharper for the lowest flow rate value $(5 \mathrm{ml} / \mathrm{h}$ for curve (c)) than at the largest ones where the influence of the heterogeneities is clearly visible (Figs. 7a and 5a). This implies that, although the density contrast is small, the ratio $\rho_{+} / \rho_{-}=1.04$ differs sufficiently from unity so that the stabilizing effect is dominant at low velocities. Then, hydrostatic pressure gradient effects induce lateral flows smoothing out the displacement fronts: similar effects were observed in stratified media with layers of different permeabilities parallel to the flow [3]. On the contrary, curves corresponding to $Q=100$ and $200 \mathrm{ml} / \mathrm{h}$ are very similar indicating that gravity is negligible (the values of $N_{\mathrm{g}}$ are, in addition, very small in both cases).

Finally we have studied the influence of the viscosity contrast by realizing similar experiments with a couple of fluids of nearly same density $\left(N_{\mathrm{g}} \cong 0\right)$ but with a viscosity ratio $M$ of order 2 (Tab. 1). Experimental concentration variation curves obtained at the same 3 distances from the inlet as for Figure 7 are displayed in Figures $8 \mathrm{a}$ and $8 \mathrm{~b}$ for flow rates respectively equal to 200 and $25 \mathrm{ml} / \mathrm{h}$. In this configuration, the viscosity contrast stabilizes the displacement front. One observes clearly at both flow rate values that the front width is much narrower than for $M=1$ at the same velocities (Figs. 5a, $7 \mathrm{a}$ and $7 \mathrm{~b}$ ). On the other hand, the two curves in Figures $8 \mathrm{a}$ and $8 \mathrm{~b}$ are much more similar than the two curves in Figures $5 \mathrm{a}$ and $7 \mathrm{~b}$ obtained at the same respective velocity values: in contrast with the case $M=1$, slope variations due to the velocity contrasts are more visible and the front width is slightly larger at the lowest flow-rate (curve (b)). This confirms that the influence of viscosity is much larger than that of gravity at the largest flow-rate values and that it is still significant for the $25 \mathrm{ml} / \mathrm{h}$ curve. 


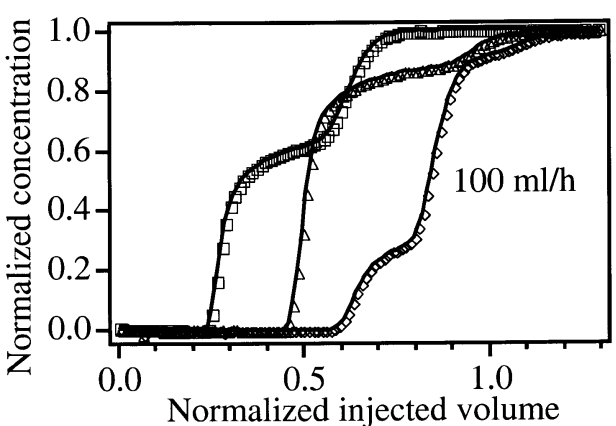

(a)

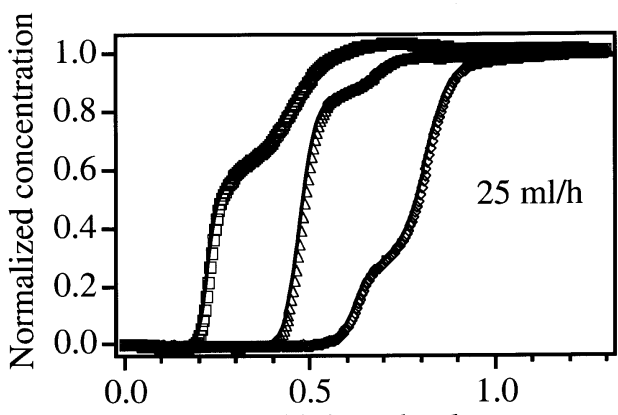

Normalized injected volume

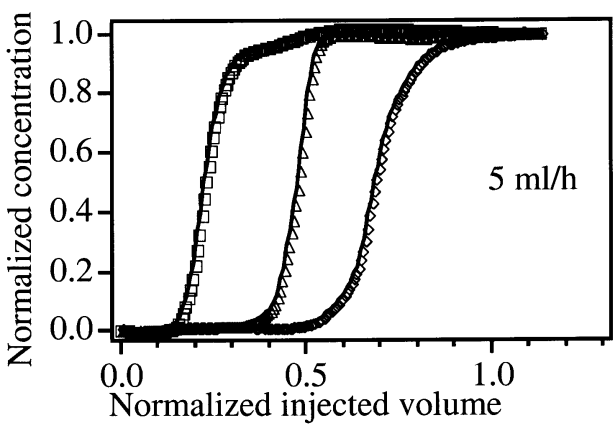

(b)

(c)

Fig. 7. Variation of the average concentration across the sample as a function of the normalized injected volume for various distances $z$ of the transducer pair from the sample inlet along the flow direction and various flow velocities $(38 \%$ sucrose solution displacing $48 \%$ glycerol solution, $M=\mu_{+} / \mu_{-}=0.98$, $\left.\rho_{+} / \rho_{-}=1.04\right)$. For each figure, the distances $z$ corresponding to given data symbols are: $(\square)$ plane \#3 $(z=52 \mathrm{~mm})$; $(\triangle) \# 8(z=127 \mathrm{~mm}) ;(\diamond) \# 12(z=187 \mathrm{~mm}) ;$ (a) flow rate $Q=100 \mathrm{ml} / \mathrm{h}\left(V=3.8 \times 10^{-2} \mathrm{~mm} / \mathrm{s}\right),(\mathrm{b}) Q=25 \mathrm{ml} / \mathrm{h}(V=$ $\left.9.5 \times 10^{-3} \mathrm{~mm} / \mathrm{s}\right),(\mathrm{c}) Q=5 \mathrm{ml} / \mathrm{h}\left(V=1.9 \times 10^{-3} \mathrm{~mm} / \mathrm{s}\right)$. The curves corresponding to $z=127 \mathrm{~mm}$ can also be compared to the data of Figure $5 \mathrm{a}$ obtained for $Q=200 \mathrm{ml} / \mathrm{h}$ $\left(V=7.6 \times 10^{-2} \mathrm{~mm} / \mathrm{s}\right)$.

Figure 8c corresponds to a displacement realized with the same couple of fluids and at the same velocity as in Figure $8 \mathrm{a}$ in the unstable configuration of the more viscous fluid displacing the lower viscosity one $(M=0.5)$. In all three measurement planes, the width of the concentration variation front is considerably larger than for a stabilizing viscosity contrast $(M=2$ in Fig. 8a) and even than for equal viscosity fluids (Figs. 5a and 7a). This increase of the width is significant even in the measurement plane closest to the injection section and a "tail" feature corresponding to viscous fluid left behind during the displacement is clearly observed.
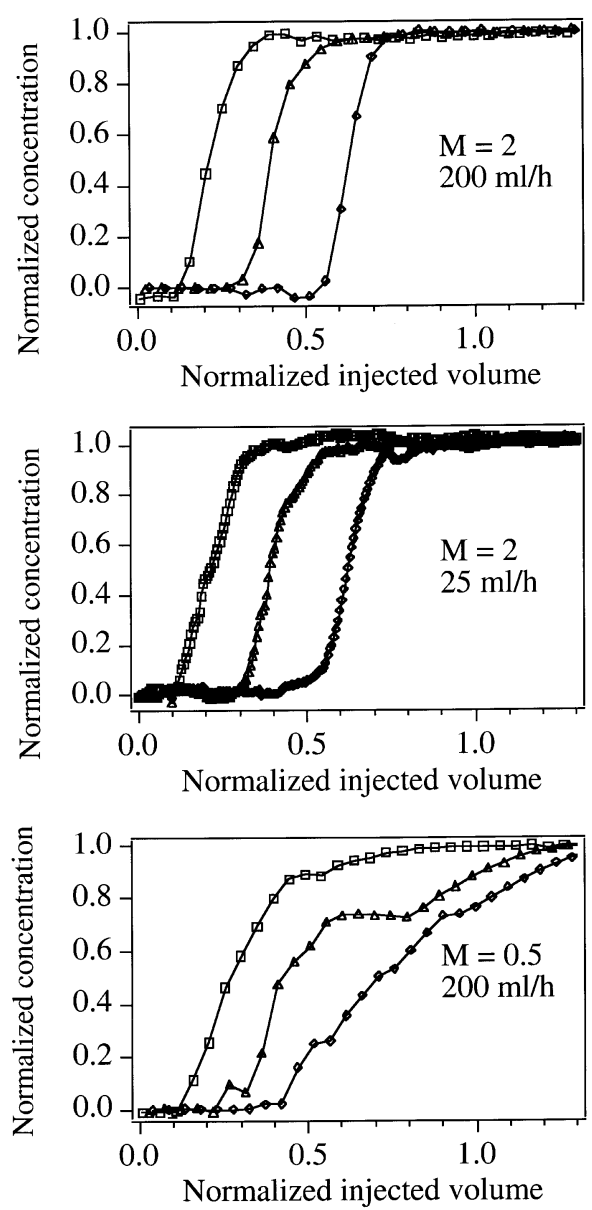

Fig. 8. Variation of the average concentration across the sample as a function of the normalized injected volume for 3 distances $z$ of the transducer pair from the sample inlet along the flow direction. For each figure, the distances $z$ corresponding to given data symbols are: $(\square)$ plane \#3 $(z=52 \mathrm{~mm})$; $(\triangle) \# 8(z=127 \mathrm{~mm}) ;(\diamond) \# 12(z=187 \mathrm{~mm})$; (a) flow rate $Q=200 \mathrm{ml} / \mathrm{h}\left(V=7.6 \times 10^{-2} \mathrm{~mm} / \mathrm{s}\right)$, (b) flow rate $Q=25 \mathrm{ml} / \mathrm{h}\left(V=9.5 \times 10^{-3} \mathrm{~mm} / \mathrm{s}\right)$. Both curves correspond to a stabilizing viscosity contrast ( $54 \%$ glycerol solution displacing $32 \%$ sucrose solution, $M=\mu_{+} / \mu_{-}=2$, $\rho_{+} / \rho_{-}=1.00$ ). (c) Flow rate $Q=200 \mathrm{ml} / \mathrm{h}$; unstable viscosity contrast (32\% sucrose solution displacing $54 \%$ glycerol solution, $\left.M=\mu_{+} / \mu_{-}=0.5, \rho_{+} / \rho_{-}=1.00\right)$.

The results obtained in 3 measurement planes are confirmed over the full sample volume by comparing the sequences of concentration images in Figures $9 \mathrm{a}$ and $9 \mathrm{~b}$ : they correspond respectively to the cases $M=2$ and $M=0.5$ and were obtained at the same flow rate value $Q=200 \mathrm{ml} / \mathrm{h}$. The front is almost flat for the case of the stabilizing viscosity contrast $M=2$ with only small distortions at the boundaries between regions of different permeabilities (Fig. 9a); on the contrary, for $M=0.5$, the invasion front clearly moves preferentially through the high permeability path (Fig. 9b) while some displaced fluid lags behind in the low permeability heterogeneities. 
(a)
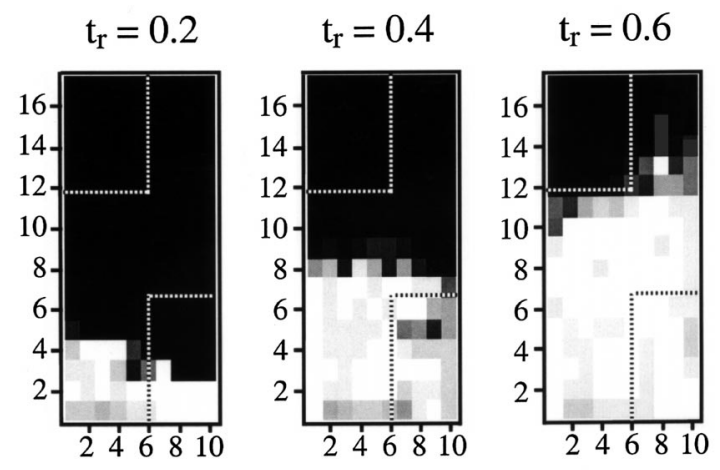

(b)

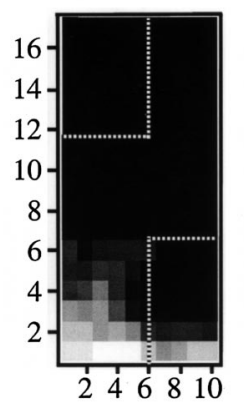

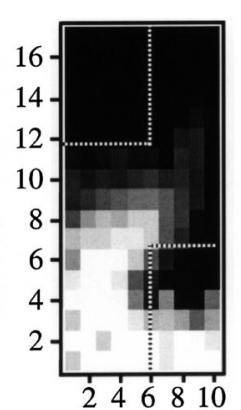

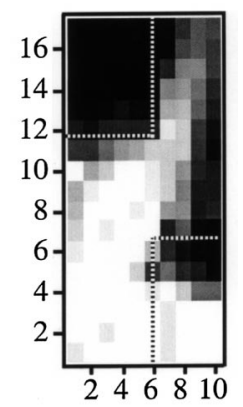

Fig. 9. Concentration variation images computed for the same experiments as Figures 8a and 8c using measurements performed at 17 equally spaced distances $z$ from the inlet section and a flow rate $Q=200 \mathrm{ml} / \mathrm{h}\left(V=7.6 \times 10^{-2} \mathrm{~mm} / \mathrm{s}\right)$. For each sequence, successive images correspond to relative injection times $t_{\mathrm{r}}=0.2, t_{\mathrm{r}}=0.4, t_{\mathrm{r}}=0.6$. The direction of fluid displacement is upwards. The plot design is the same as in Figure 3. (a) Stable viscosity contrast: $54 \%$ glycerol solution displacing $32 \%$ sucrose solution $\left(M=\mu_{+} / \mu_{-}=2, \rho_{+} / \rho_{-}=1.00\right)$. (b) Unstable viscosity contrast $(32 \%$ sucrose solution displacing $54 \%$ glycerol solution, $\left.M=\mu_{+} / \mu_{-}=0.5, \rho_{+} / \rho_{-}=1.00\right)$.

The sweep efficiency of the displacement is therefore considerably better in the stabilizing case.

Let us finally note that these effects of viscosity might be equally well-reproduced in the Hele-Shaw cell models. The effect of the density contrasts might also be modelled (at the cost of a more complex inclinable optical set-up) and even adjusted by modifying continuously the inclination angle of the cell.

\section{Lattice Boltzmann numerical simulations}

Experiments presented in the two previous parts have allowed to analyze in detail the spreading of a miscible fluid displacement front due to permeability heterogeneities of simple geometry. Due to experimental constraints, such measurements can only be performed for a few values of the parameters characterizing the contrast between fluid properties. In order to generalize these results in future work to other fluid couples (and other geometries), we plan to realize in some chosen geometries a set of numerical simulations using a lattice Boltzmann algorithm [23-29]. We shall now in a first step determine whether this

type of simulations can reproduce accurately the above experimental results obtained either in the Hele-Shaw geometry or for 3D bead packings.

\subsection{Lattice Boltzmann simulation algorithm}

In the kinetic gas theory, simple collisions, preserving mass and momentum, are able to generate gas transport properties such as viscosity, mass and thermal diffusion. Statistical physics provide a more elaborate description of the fluid state by taking into account multiple collisions and interactions. The essence of lattice gas modelling is to implement this statistical physics approach on a lattice with a large enough number of degrees of symmetry and freedom. Then, the macroscopic behaviour of the fluids will be derived from a microscopic description of the (quasi-) particles trajectories and collisions. Thus, unlike classical discretization methods (finite-elements, finite-volumes), lattice gases are not solvers but generators of the NavierStokes equation.

Since the pioneering work of Frisch, Hasslacher and Pomeau [23], who demonstrated the ability of a lattice gas to develop macroscopically a behavior very close to Navier-Stokes equations, an extended literature has been available [24]. Applying the statistical physics ideas of Bhatnagar, Gross and Krook [25], Qian et al. [26] have developed the lattice BGK technique for one fluid, where the collision step is reduced to a multiplication by a relaxation parameter. The viscosity of the fluid can therefore easily be tuned, since one has only to fix the relaxation parameter of the evolution equation for the mass density, at the beginning of the simulation.

We use the so-called 2DQ9 model [26] to describe the fluid dynamics in 2D. Basically, occupation probabilities for the particles are distributed on the nodes of a square grid, pointing towards eight directions (four medians and four diagonals), plus one corresponding to rest. They collide at each time step and propagate following a Boltzmann relaxation equation. The variable for the flow is $N_{i}(\mathbf{r}, t)$; it represents the mean occupation number of particles in direction $i$ at node $\mathbf{r}$ and time $t$. This number is updated at each time step using the relation:

$$
N_{i}\left(\mathbf{r}+\mathbf{e}_{i}, t+t_{0}\right)=(1-\omega) N_{i}(\mathbf{r}, t)+\omega N_{i}^{\mathrm{eq}}(\mathbf{r}, t)
$$

where $t_{0}$ is the time step, $\mathbf{e}_{i}$ denotes the direction vector connecting $\mathbf{r}$ to the neighbors, and $\omega$ is the relaxation parameter ranging between 0 and 2 . The equilibrium distribution $N_{i}^{\text {eq }}$ is given by

$N_{i}^{\mathrm{eq}}(\mathbf{r}, t)=t_{i} \rho\left[1+c_{\mathrm{s}}^{-2} \mathbf{V} \cdot \mathbf{e}_{i}+c_{\mathrm{s}}^{-2} \frac{V_{\alpha} V_{\beta}}{2}\left(c_{\mathrm{s}}^{-2} e_{i \alpha} e_{i \beta}-\delta_{\alpha \beta}\right)\right]$

where $\alpha, \beta$ are the Cartesian coordinates, $c_{\mathrm{s}}=1 / \sqrt{3}$ is the sound velocity, and $t_{i}$ takes the values $1 / 9$ and $1 / 36$ for the median and diagonal directions, respectively, and $4 / 9$ for rest. 
The mass and momentum density per site are determined by the relations $\rho=\sum_{i} N_{i}$ and $\rho \mathbf{V}=\sum_{i} N_{i} \mathbf{e}_{i}$. This model leads to the Newtonian incompressible NavierStokes equation (for $V \ll c_{\mathrm{s}}$ ), with a kinematic viscosity $\nu$ linked to the relaxation parameter $\omega$ through

$$
\nu=\frac{1}{3}\left(\frac{1}{\omega}-0.5\right) .
$$

It can be shown [27] that the effect of an external force $\mathbf{F}$ can be accounted for by replacing, at each time step, the velocity $\mathbf{V}$ in the equilibrium distribution (7) by $\mathbf{V}+(\tau / \rho) \mathbf{F}$ where $\tau=1 / \omega$ is the relaxation time due to collisions. This amounts to introduce in the Navier-Stokes equation a body force $\mathbf{F}$ so that:

$$
\rho\left[\frac{\partial \mathbf{V}}{\partial t}+(\mathbf{V} \cdot \operatorname{grad}) \mathbf{V}\right]=-\operatorname{grad} p+\eta \Delta \mathbf{V}+\mathbf{F} .
$$

In order to take into account the effect of Darcy pressure gradients due to viscous friction in the pores, we follow the procedure described above and use a body force $\mathbf{F}=-\eta \mathbf{V}(\mathbf{r}) / k(\mathbf{r})$ where $k(\mathbf{r})$ is the permeability at site $\mathbf{r}$. This leads, in the stationary, low Reynolds number limit to the equation:

$$
0=-\operatorname{grad} p-\frac{\eta}{k} \mathbf{V}+\eta \Delta \mathbf{V}
$$

The first and last term on the right hand side of the equation correspond to Stokes equation which reflects a balance between the pressure gradient and the Laplacian viscous term $\eta \Delta \mathbf{V}$. The effect of Darcy's pressure drop is represented by the additional - body force like term $-\eta \mathbf{V} / k$. In an homogeneous medium, the Laplacian term vanishes: the equation then reduces to Darcy's law: $\operatorname{grad} p=-(\eta / k) \mathbf{V}$. We shall call equation (10) DarcyStokes: the simultaneous inclusion of both the Darcy and the Laplacian terms allows to smooth out exceedingly sharp variations of the velocity field which appear when large permeability gradients are present. In such cases, introducing Stokes term spreads out the gradients over a boundary layer of typical thickness $\sqrt{k}$. Equation (10) thus allows in particular to deal with the effect of discontinuities between different permeability zones.

The modelization of the displacement of a fluid by another miscible one or of the dispersion of a passive tracer requires to solve a transport equation for the scalar $C(\mathbf{r}, t)$ representing the local concentration of one of the fluids or the tracer concentration. The variation of $C(\mathbf{r}, t)$ can be computed in a similar way as above by solving the convection diffusion equation [28,29]:

$$
\frac{\partial C}{\partial t}+\mathbf{V} \cdot \operatorname{grad} C=D_{\mathrm{m}} \Delta C
$$

where the molecular diffusion coefficient $D_{\mathrm{m}}$ is related to the relaxation parameter of Boltzmann's equation for the scalar $C$. Additional details on the algorithm and simulation scheme described here can be found in reference [30].

This extension of the BGK method to describe the transport of scalar quantities has been successfully tested [30] in the case of the dispersion of a passive tracer in a laminar flow field [10]. When the tracer concentration influences the fluid properties (as in the present case where the two fluids may have different viscosities and densities) the physical parameters of the mixture vary as mixing takes place. We need therefore to introduce in the simulation a mixing rule for the fluids: we assume in particular that the local viscosity $\eta(C)$ varies exponentially with the concentration $C$. With such a procedure, the Darcy-Stokes equation and the convection-diffusion equation are coupled through the concentration-dependent viscosity: the velocity field and the concentration depend then on each other. Let us note that this scheme can be applied to nonNewtonian fluids as well; then, the effective kinematic viscosity input into the relaxation equation becomes a function of the local shear rate [31]. Our simulation results can be compared to the measurements in actual experiments by assuming that time scales as the time step, length as the unit lattice size. Thus velocity can be obtained from a number of mesh sizes representing the mean displacement per time step, the density is the mass per square unit lattice size, etc. The lattice size in the simulations is $x \times y=44 \times 400$. Usable viscosity and diffusion coefficient values range between $10^{-4}$ and $10^{-1}$.

The characteristic dimensionless numbers of our problem are the ratio $M=\rho_{-} / \rho_{+}$of the respective viscosities of the displaced and injected fluid and the Peclet number, defined by $P e=U H / D_{\mathrm{m}}$, where $H$ is the extent of the injected fluid in the flow direction. The Peclet number is the ratio of the transverse diffusion time to the advection one. When $P e$ is much greater than one, molecular diffusion becomes negligible and the diffusion of the injected fluid during the transit time is almost negligible. Finally, the Reynolds number is low enough so that one can almost always assume that Stokes equation is valid $(R e \ll 1)$.

\subsection{Numerical simulation results}

The Péclet number value in the simulation is 250 implying that diffusion is almost negligible and that the interface between the two fluids is well-defined. Figures 10a-10d display the numerical simulations with the same presentation as for the experimental results in Figure 3. Both the viscosities and densities of the two fluids have the same values and the ratio between permeabilities in the high and low permeability regions is equal to 4 . Figure $10 \mathrm{a}$ displays the geometry of the invasion front at several equally spaced times; Figure 10b the streamlines. Figure 10c corresponds to a superposition of the two previous sets of curves and Figure 10d presents the velocity vector field. In all cases, these results are qualitatively very similar to the experimental observations of Figures $3 \mathrm{a}-3 \mathrm{~d}$. Let us note however that the velocity contrast between the high and low velocity regions in the center part of the field of view is somewhat larger in the simulation as in the experiment. As already noted previously, this is due to the fact that, in the Hele-Shaw cell, the local permeability is adjusted by changing the local thickness and therefore the effective "porosity". A variation of the permeability by a factor 
(a)

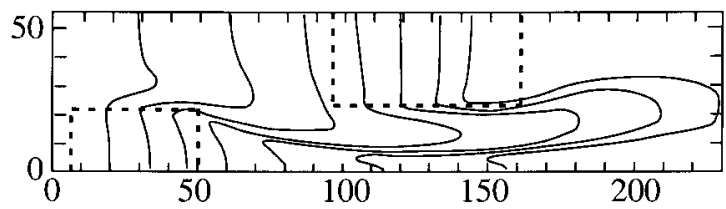

(b)

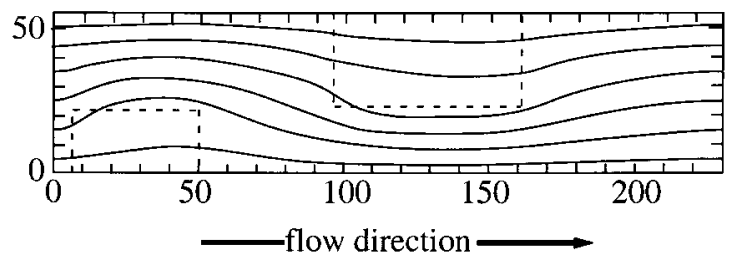

(c)

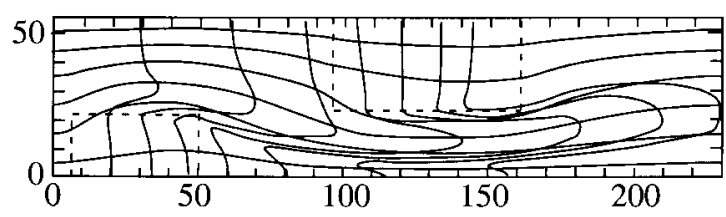

(d)

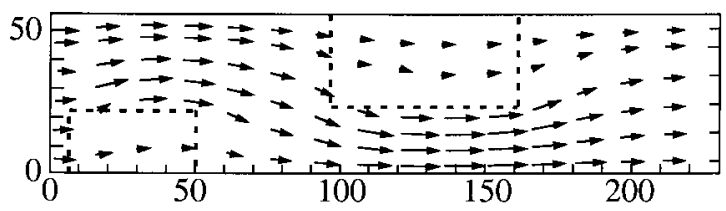

Fig. 10. Numerical simulation of fluid displacement in a $2 \mathrm{D}$ porous medium with large heterogeneities of same geometry as Figures 1 and 4 and for an equivalent Péclet number of 200. (a) Sequence of invasion fronts obtained at regular time intervals $\Delta t$; (b) flow lines; (c) superposition of invasion fronts and flow lines; (d) flow velocity vector field.

of 4 implies a corresponding variation of the thickness and the porosity by a factor of 2 . This local decrease of the porosity in the Hele-Shaw cell correspondingly increases velocity due to mass flow rate conservation. This porosity reduction effect is not taken in account in the simulations of Figure 10.

We have also compared the simulation results to acoustic measurements performed on the 3D system. Figure 11 displays experimental and numerical mean concentration variations obtained at the same 3 distances from the inlet section as in Figures 7 and 8. While the main features of the different curves are well-reproduced, sharper variations are observed in the numerical curves as in the experimental ones: this may be due to the fact that local geometrical dispersion is not included in the simulation while - as indicated above - it is present in the grain packing. A second possible origin of this discrepancy is the finite $(1 \mathrm{~cm})$ spatial resolution of the acoustic transducers in the vertical direction which also smoothen sharp variations.

We can thus conclude that the lattice Boltzmann simulations reported above reproduce satisfactorily the key features of the invasion front displacements in the experiments reported in parts 2 and 3 of the present work. The agreement might be improved - particularly for the bead packings - by fine tuning the different porosity and permeability values used in the simulation and taking into

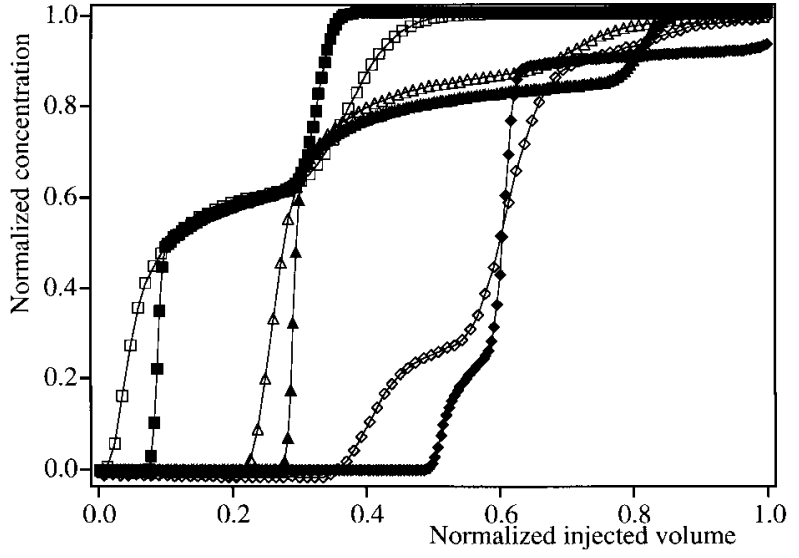

Fig. 11. Numerical and experimental (acoustical measurements) average concentration variation at 3 distances $z$ from the inlet $(\square$ and $\square)$ plane \#3 $(z=52 \mathrm{~mm}) ;(\triangle$ and $\boldsymbol{\Delta}) \# 8$ $(z=127 \mathrm{~mm}) ;(\diamond$ and $\diamond) \# 12(z=187 \mathrm{~mm})$. Open symbols correspond to the experiment and dark symbols to the simulations. Flow rate $Q=100 \mathrm{ml} / \mathrm{h}\left(V=3.8 \times 10^{-2} \mathrm{~mm} / \mathrm{s}\right)$. $M=\mu_{+} / \mu_{-}=1, \rho_{+} / \rho_{-}=1.00$ for the simulation, 1.04 for the experiment.

account their correlations. On the other hand, taking into account Taylor dispersion would be required to obtain results fully comparable with Hele-Shaw experiments. This could be achieved through 3D simulations with same aspect ratios as the experiment and might be a useful tool for other problems such as tracer dispersion in fractures with spatially variable apertures. Since Taylor dispersion is not significant in porous media relevant to the present work, the $2 \mathrm{D}$ simulations discussed here are better adapted to our purpose. They are much less computer demanding and allow to repeat computations for many different fluid parameter values and heterogeneity distributions.

\section{Conclusion}

In the present paper, we demonstrated how a full picture of fluid displacements in heterogeneous media and of their dependence on fluid parameters can be obtained from Hele-Shaw and acoustic scanner experiments and lattice Boltzmann numerical simulations.

Hele-Shaw cell models allow for high resolution mappings of the front geometry and of its time variations. Moreover, by combining images of the front at regular time intervals and images of the flow lines realized by pointlike dye injections, one obtains directly a map of the flow velocity field. It should also be possible to model the effect of gravity and of stabilizing or destabilizing viscosity contrasts by using vertical or obliquely inclined cells and fluids of different densities and viscosities.

Acoustic scanner techniques, on the other hand, use measurements of the mean sound velocity along selected paths across the medium to determine the respective 
concentrations of the injected and displaced fluid. In the case of a bidimensional permeability distribution, one obtains directly a low resolution (typ. $10 \times 17$ ) "acoustic image" of the displacement front and of its time dependence. This technique can also be applied to arbitrary three-dimensional permeability distributions. Although a full 3D image cannot be determined, one can still obtain important informations on the width of the displacement front and on its structure. Comparing concentration variations with time along different flow paths will for instance help detect and map preferential flow paths. The dependence of the front on time, on distance from the inlet section, on flow rate and on fluid characteristics can also be studied.

The two experimental approaches are therefore complementary. The acoustic technique can be applied to 3D opaque materials and natural rocks and take into account easily the effect of density; on the other hand $2 \mathrm{D}$ models give high resolution pictures of the displacement even when the injected and displaced fluid have identical physical properties (acoustical measurements require a sound velocity contrast).

Finally the two types of experiments can be usefully associated to 2D Boltzmann lattice computations taking into account both the Darcy pressure gradient and a Stokes diffusion term (in order to deal with permeability discontinuities). One particularly helpful feature of this technique is its flexibility which allows to change easily the fluid properties, the flow geometry and the permeability distribution. It is also possible in such simulations to introduce the effect of local geometrical dispersion in the 3D medium as a diffusive process of suitable characteristics. After such simulations have been validated experimentally in a given geometry for a few fluid couples, they can be run for many different sets of fluid parameter values. It is also possible to study easily how variations of the size and permeability of specific parts of the medium influence concentration variations detected in selected sections. This represents a first step before dealing with the inverse problem of the estimations of spatial probability distributions from concentration variation measurements realized at a few selected points.

This work has been partly funded by the Ecodev (ARC Géothermie des Roches Fracturées) and PRNH-CNRS Programs, by the Groupement de Recherches "Physique des Milieux Hétérogènes Complexes" and by NATO grant \# CRG 973 049. We thank G. Chauvin, C. Frenois and C. Saurine for their help in the realization of the experimental set-up, B. Goyeau, B. Guerrier, J. Liu, J. Martin and Y. Yortsos for useful discussions and H. Bockzmak for his contribution to the experiments.

\section{References}

1. J. Bear, Dynamics of fluids in porous media (Elsevier Publishing Co., New-York, 1972).

2. F.A.L. Dullien, Porous media, fluid transport and pore structure, 2nd edn. (Academic Press, New-York, 1991).

3. C. Leroy, J.P. Hulin, R. Lenormand, J. Contamin. Hydrol. 11, 51 (1992).

4. R. Lenormand, Transp. Porous Media 18, 245 (1995); R. Lenormand, B. Wang, Transp. Porous Media 18, 2263 (1995).

5. J.R. Waggoner, J.L. Castillo, L.W. Lake, paper SPE 21237 (1991).

6. K.S. Sorbie, G.E. Pickup, P.S. Ringrose, J.L. Jensen, paper SPE/DOE 24140 (1992).

7. L.W. Gelhar, C.A. Axness, Water Resources Res. 19, 161 (1983).

8. G. Dagan, Water Resources Res. 26, 1281 (1990).

9. L. Zhan, Ph.D. thesis, University of Southern California, 1999; Y.C. Yortsos (private communication).

10. G.I. Taylor, Proc. Roy. Soc. A 219, 186 (1953).

11. R. Aris, Proc. Roy. Soc. A 235, 65 (1956).

12. Y.C. Yortsos, Transp. Porous Media 18, 107 (1995).

13. Z.M. Yang, Y.C. Yortsos, Phys. Fluids 9, 286 (1997).

14. D. Salin, W. Schon, J. Phys. Lett. 42, L477 (1981).

15. J.-C. Bacri, M. Hoyos, R. Lenormand, N. Rakotomalala, A. Soucemariadin, D. Salin, J. Phys. III France 1, 1455 (1991).

16. P.G. Saffman, J. Fluid Mech. 6, 321 (1959); ibid. 7, 194 (1960).

17. J.J. Fried, M. Combarnous, Adv. Hydrosci. 7, 169 (1971).

18. D.L. Koch, J.F. Brady, J. Fluid. Mech. 154, 399 (1985).

19. L.J.T.M. Kempers, H. Haas, J. Fluids Mech. 267, 299 (1994).

20. J.-C. Bacri, D. Salin, R. Woumeni, Phys. Rev. Lett. 67, 2005 (1991).

21. H.A. Tchelepi, F.M. Orr, N. Rakotomalala, D. Salin, R. Woumeni, Phys. Fluids A 5, 1558 (1993) and references therein.

22. D. Loggia, N. Rakotomalala, D. Salin, Y.C. Yortsos, Europhys. Lett. 32, 633 (1995).

23. U. Frisch, B. Hasslacher, Y. Pomeau, Phys. Rev. Lett 56, 1505 (1986).

24. D.H. Rothman, S. Zaleski, Rev. Mod. Phys. 66, 1417 (1994) and references therein.

25. P.L. Bathnagar, E.P. Gross, M. Krook, Phys. Rev. 94, 511 (1954).

26. Y.H. Qian, D. d'Humières, P. Lallemand, Europhys. Lett. 17, 479 (1992).

27. H. Chen, Comp. Phys. 7, 632 (1993)

28. E.G. Flekkoy, Phys. Rev. E 47, 4247 (1993).

29. U. Oxaal, E.G. Flekkoy, J. Feder, Phys. Rev. Lett. 72, 3514 (1994).

30. N. Rakotomalala, D. Salin, P. Watzky, J. Fluid Mech. 338, 277 (1997).

31. N. Rakotomalala, D. Salin, P. Watzky, Phys. Fluids 8, 3200 (1996). 